\title{
The Use of Botulinium Toxin as a Nonsurgical Adjuvant in the Closed Management of Condylar/Subcondylar Fractures
}

\author{
NIVEEN FATHY AL-MAHMOUDY, M.D.*; ALLAM EL-SAYED ALLAM, M.D.** and \\ AMIR EL-BARBARY, M.D.* \\ The Departments of Plastic Surgery* and Radiodiagnosis**, Faculty of Medicine, Ain Shams University, Cairo
}

\begin{abstract}
Background: Mandibular condylar fractures are common, with prevalence between $25 \%$ and $35 \%$ of all mandibular fractures. In children, the condylar fractures are usually managed with maxilla-mandibular fixation, where as in adults the treatment of condylar fractures remains controversial. Several investigators validated the use of Botulinium Toxin (BTX) for treatment of recurrent temporomandibular joint dislocation. However, there are very limited studies that assessed (BTX) injection in the management of condylar/ subcondylar fractures.
\end{abstract}

Patients and Methods: Ten patients presenting with condylar/subcondylar fractures either isolated or associated with other mandibular fracture were treated by Ultrasound guided (BTX) injection to Lateral Pterygoid Muscle (LPM) followed by application of arch bar and guiding elastics 2-3 days later.

Results: Patients retained good occlusion with unrestricted mouth opening. Minor deviation upon mouth opening was observed in some of the cases. Good alignment of the condylar /subcondylar fracture was documented radiologically over a minimum of three-month follow-up period.

Conclusion: The use of botulinium toxin in condylar fractures is safe, and can be used as nonsurgical adjuvant in management of condylar/subcondylar fractures.

Key Words: Condylar/subcondylar fracture - Botulinium toxin.

\section{INTRODUCTION}

Treatment of condylar fractures has been a controversial topic. Four main treatment modalities have been described in the literature: Mandibular physical therapy consisting of ranging exercises without maxillomandibular fixation, a short period of maxillomandibular fixation followed by mandibular physical therapy, open reduction followed by maxilla-mandibular fixation and open reduction and internal fixation of the condylar fracture [1].

Mandibular condylar fractures are common, with prevalence between $25 \%$ to $35 \%$ of all mandibular fractures $[2,3]$. They are usually managed with maxilla-mandibular fixation, in children and their treatment protocols remain debatable in the adult [4]. Despite the discussion of absolute and relative indications of open reduction for managing mandibular condylar fractures in the literature [5], there are no studies providing evidence-based absolute superiority of open reduction over closed treatment [6]. Treatment of condylar fractures appears to be basically set on practice and experience $[6,7]$.

The clinical use of Botulinum Toxin (BTX) has expanded during the last ten years. It is used in the treatment of severe bruxism, facial muscle spasm, hypertrophy of the masticatory muscles and dystonias [8], myofascial pain syndrome [9], temporomandibular disorders [10], chronic migraine [11] and Frey's syndrome [12].

In 1997, Daelenetal [13], first described (BTX) injection into the Lateral Pterygoid Muscle (LPM) for the treatment of habitual temporomandibular joint dislocation. Several investigators subsequently reported using (BTX) therapy for recurrent temporomandibular joint dislocation. They reported that it was effective and minimal unfavorable reactions were observed [14-16]. The use of (BTX) injection in the management of condylar/subcondylar fractures is limited to only one report in the literature [17] with insufficient information concerning related complications and the detailed application of the treatment.

The aim of this study is to assess the effect of (BTX) injection to the lateral pterygoid muscle with ultrasound guidance as a nonsurgical adjuvant in cases of condylar/subcondylar fracture.

\section{PATIENTS AND METHODS}

This study was done in Ain-Shams University hospitals from April 2017 to September 2018. 
Patients presenting to the ER with condylar/ subcondylar fractures with or without other mandibular fracture(s) were included while excluding patients with major poly-trauma affecting other systems or major medical comorbidity. In addition, patients with relative contraindications to (BTX) injection were excluded such as pregnancy, lactation, neuromuscular diseases (e.g., myasthenia gravis), motor neuron disease, allergy to any of the components of (BTX) (i.e. human albumin), infection at injection site and psychologically unstable patients.

Patients were carefully assessed \& examined for:

- Occlusion assessment (1: Same to pretraumatic, 2: Minor difference, 3: Functional malocclusion, 4: Needs occlusal correction, 5: Gross malocclusion) [18].

- Pain with a visual analog scale assessment: Values from 0 (no pain) to 10 (strongest pain) [18].

- Range of motion: By the mouth opening (maximum inter-incisal distance) [18].

- Deviation of mandible on mouth opening [18].

All patients were photographed for occlusion and mouth opening. Full laboratory investigations and radiological evaluation (panorama \& CT facial; coronal, axial \& 3D) were done.

Botulinum toxin A (10-20 IU) was first injected into LPM 2-3 days prior to any surgical intervention (for the BTX to be clinically effective). The procedure was performed using $25 \mathrm{~g}$ needle under ultrasound guidance (MYLAB 25-Esaote S.P.A, Italy) with $6 \mathrm{MHz}$ linar transducer. The LPM was identified below the zygomatic arch deep to the master muscle in the mandibular notch between the coronoid process and condyle of the mandible. 10-20 units of (BTX) were injected by free hand technique in transverse view where the needle appears as echogenic dot inside the muscle with care not to injure maxillary artery nearby Fig. (1).

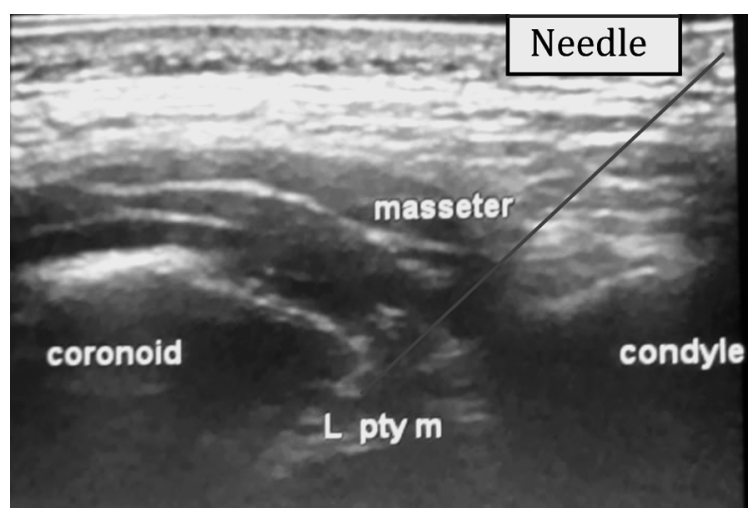

Fig. (1): Ultrasound guided injection of (BTX) in LPM.
All patients received general anesthesia, with nasal intubation, followed by application of guiding elastic bands over upper and lower arch bars. Open reduction and internal fixation by 2.3 plates and screws were carried out to other concomitant fractures. The patients were discharged one to two days after the procedure and remained on a soft diet for a month thereafter.

All patients were followed-up twice a week in the first two weeks to verify the occlusion and ensure adequate performance of functional rehabilitation exercises. Guiding elastics were removed once satisfactory occlusion was achieved and the frequency of future visits was based accordingly over the following three months.

Post-operative CT was obtained immediately and after three months. Photographs were taken for all patients to document occlusion and mouth opening. Any possible complications encountered along the active treatment phase were recorded.

\section{RESULTS}

Ten patients with subcondylar fractures or fractures at the condylar neck were included in this study ( 8 males \& 2 females) with age ranging between 7-44 years, two patients had isolated unilateral condylar/subcondylar fracture, two patients had bilateral condylar fracture associated with other mandibular fractures, six patients had unilateral condylar/subcondylar fracture associated with other mandibular fractures (Table 1).

Occlusion was obtained intraoperatively in all cases and maintained through the use of elastic guiding for one or two weeks then the patients were followed-up regularly. Mouth opening was limited at the beginning with progressive improvement and patients were able to admit 2.5 fingers at the end of two months. Minor deviation was noticed upon mouth opening in some cases. Pain threshold decreased by time reaching score 1 (least pain) by four weeks.

The fractured condylar process or subcondylar segment were found to come in good approximation and continue in reduced positions in most of the patients as shown in post-operative CT Figs. $(2,3)$. Only one patient developed an anterior open bite and cross bite on the right side that was referred for correction by orthodontics cases number 2 (Table 2). 
Table (1): Demographic data of the patients.

\begin{tabular}{lllll}
\hline No. & Sex & Age & Mode of trauma & Fracture site \\
\hline 1 & M & 7 & MCA & LT subcondylar fracture \\
2 & M & 33 & MCA & RT subcondylar fracture with LT parasymphyseal \\
3 & F & 27 & Falling from height & RT condylar fracture LT subcondylar with alveolar fracture \\
4 & M & 38 & MCA & Lt condylar fracture \\
5 & M & 36 & MCA & Bilateral condylar fracture with LT parasymphyseal \\
6 & F & 30 & MCA & RT subcondylar with LT anterior body fracture \\
7 & M & 44 & Falling from height & RT subcondylar LT condylar fractures \\
8 & M & 39 & Fight & LT subcondylar with RT parasympyseal fracture \\
9 & M & 30 & MCA & Bilateral condylar fracture with symphyseal fracture \\
10 & M & 33 & MCA & LT subcondylar with RT parasymphyseal \\
\hline
\end{tabular}

Table (2): Pre-operative and post-operative assessment.

\begin{tabular}{|c|c|c|c|c|c|c|c|c|}
\hline & \multicolumn{2}{|c|}{ Occlusion } & \multicolumn{2}{|r|}{ Pain } & \multicolumn{2}{|c|}{ Mouth opening $(\mathrm{cm})$} & \multicolumn{2}{|c|}{ Mouth deviation } \\
\hline & Pre-op & 3 month post-op & Pre-op & 3 month post-op & Pre-op & 3 month post-op & Pre-op & 3 month post-op \\
\hline 1 & 2 & 1 & 8 & 2 & Limited & $3 \mathrm{~cm}$ & Cross bite & Minor deviation \\
\hline 2 & 5 & 4 & 6 & 3 & Limited & $4 \mathrm{~cm}$ & Cross bite & Major deviation \\
\hline 3 & 5 & 1 & 9 & 4 & Limited & $4.5 \mathrm{~cm}$ & Cross bite & Minor deviation \\
\hline 4 & 3 & 1 & 6 & 1 & Limited & $4.5 \mathrm{~cm}$ & No mouth deviation & No mouth deviation \\
\hline 5 & 5 & 2 & 5 & 2 & Limited & $4.5 \mathrm{~cm}$ & No mouth deviation & No mouth deviation \\
\hline 6 & 5 & 1 & 9 & 3 & Limited & $4 \mathrm{~cm}$ & Cross bite & Minor deviation \\
\hline 7 & 5 & 1 & 7 & 1 & Limited & $4.5 \mathrm{~cm}$ & Cross bite & Minor deviation \\
\hline 8 & 5 & 1 & 6 & 3 & Limited & $4 \mathrm{~cm}$ & Cross bite & Minor deviation \\
\hline 9 & 5 & 2 & 7 & 2 & Limited & $4.5 \mathrm{~cm}$ & No mouth deviation & No mouth deviation \\
\hline 10 & 5 & 1 & 8 & 1 & Limited & $4.5 \mathrm{~cm}$ & Cross bite & Minor deviation \\
\hline
\end{tabular}

(A)

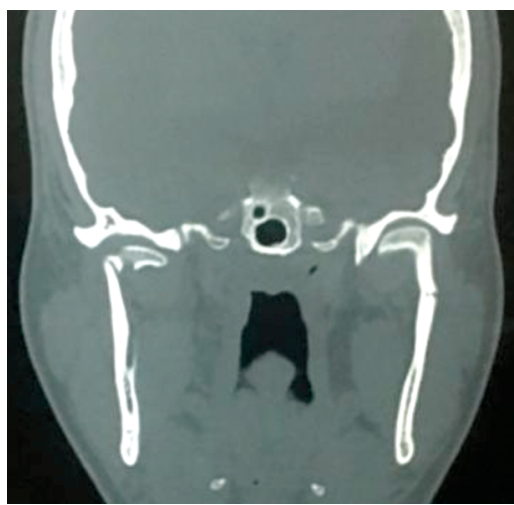

(B)

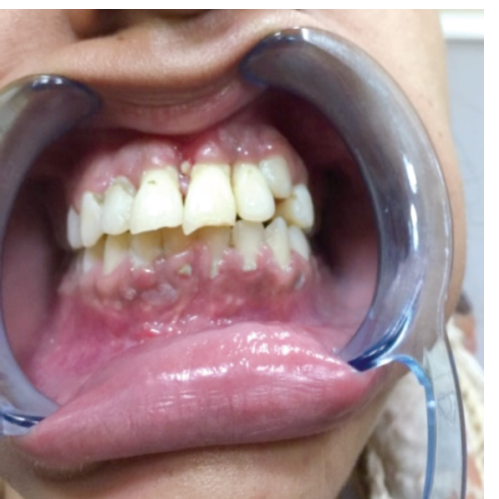

(C)

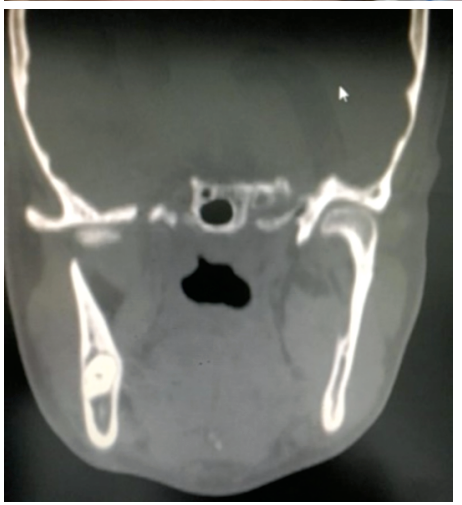

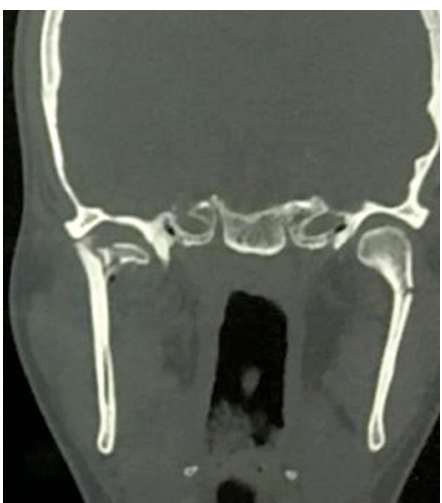
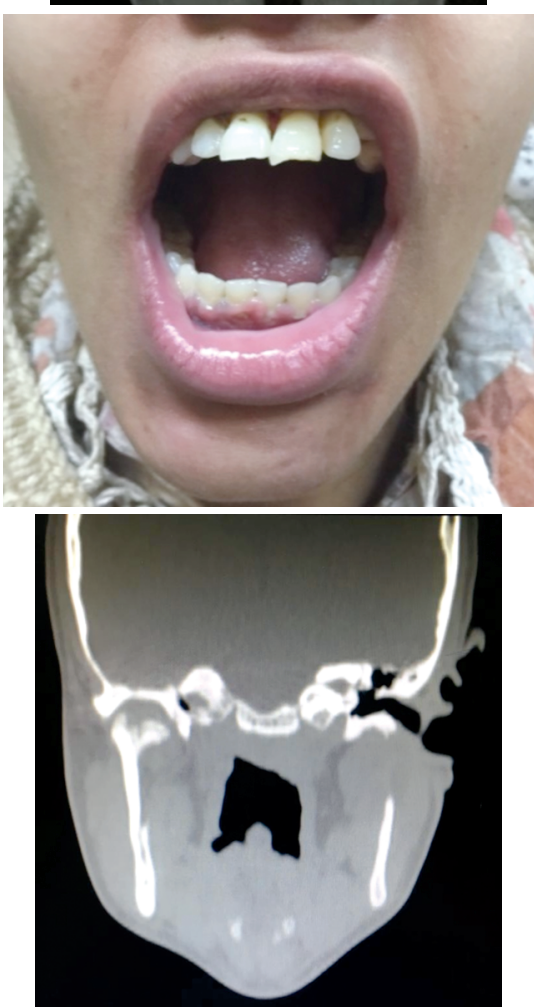

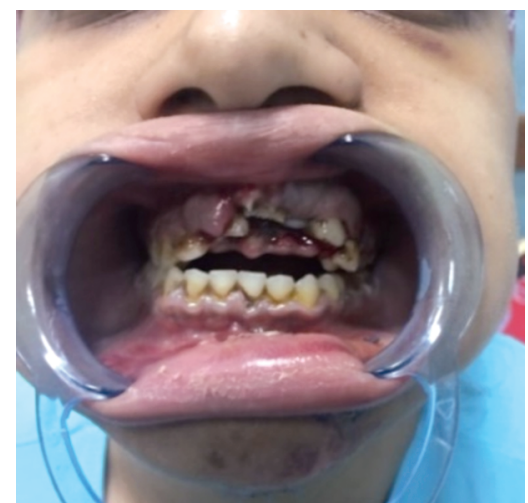

Fig. (2): A- CT facial (coronal view) of a 33 year old female patient with RT condylar and Lt subcondylar fracture, with alveolar fracture, B- 3 months postoperative, patient had good occlusion and unrestricted mouth opening, C- CT facial (coronal view) 3 months post-operative showing good alignment of fracture and unrestricted mouth opening in the presence of minor deviation. 
(A)
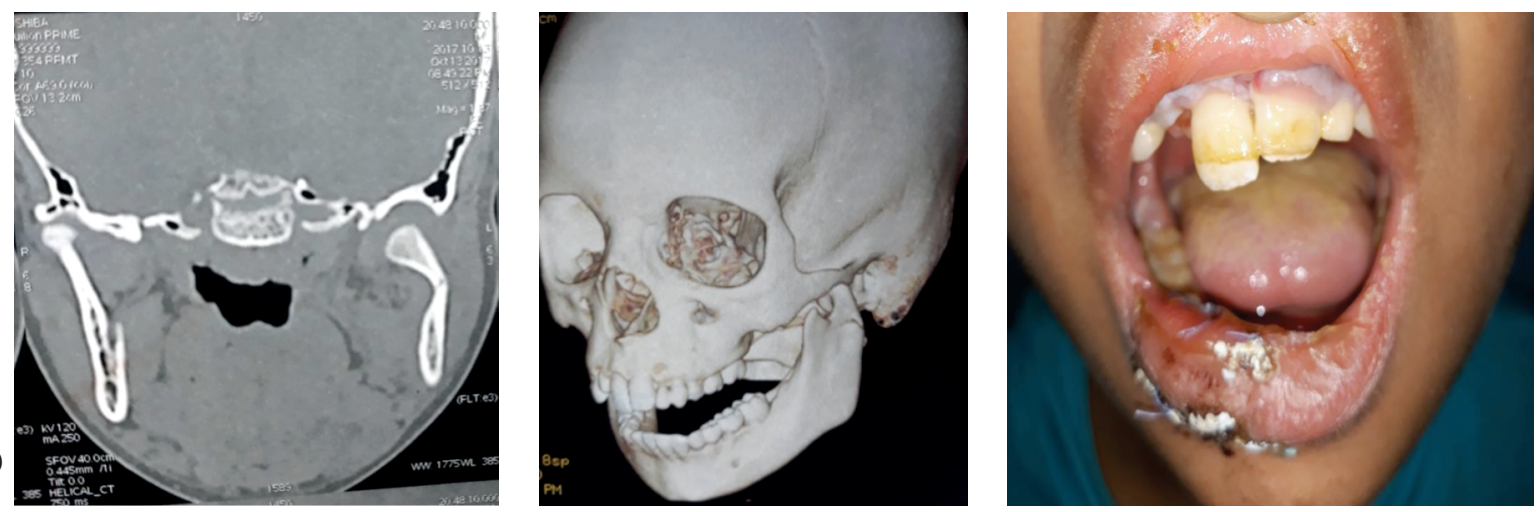

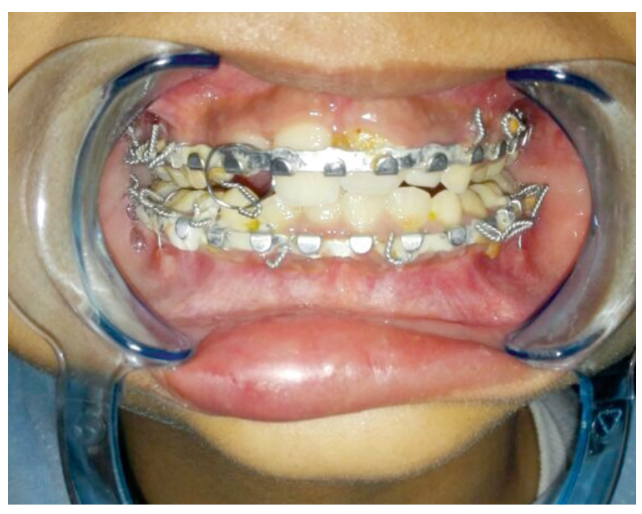

(B)

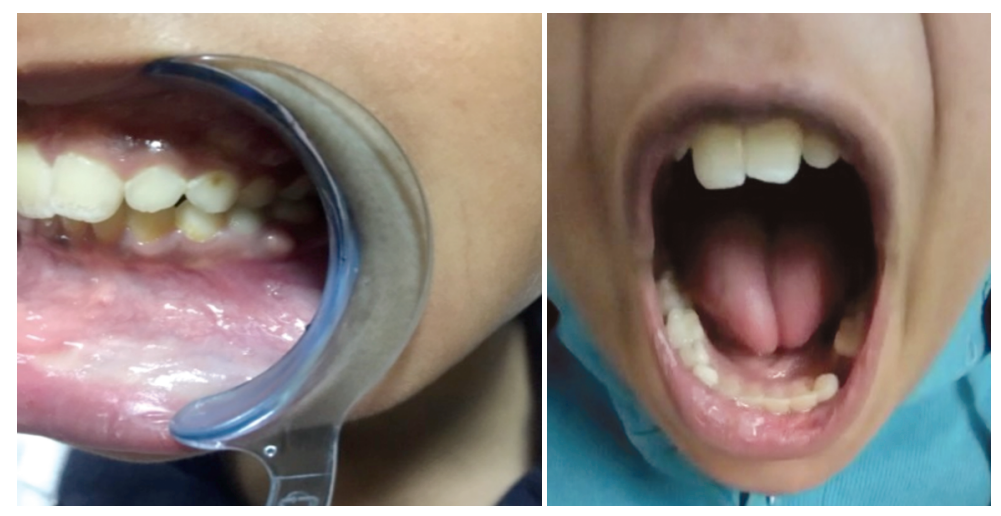

(C)
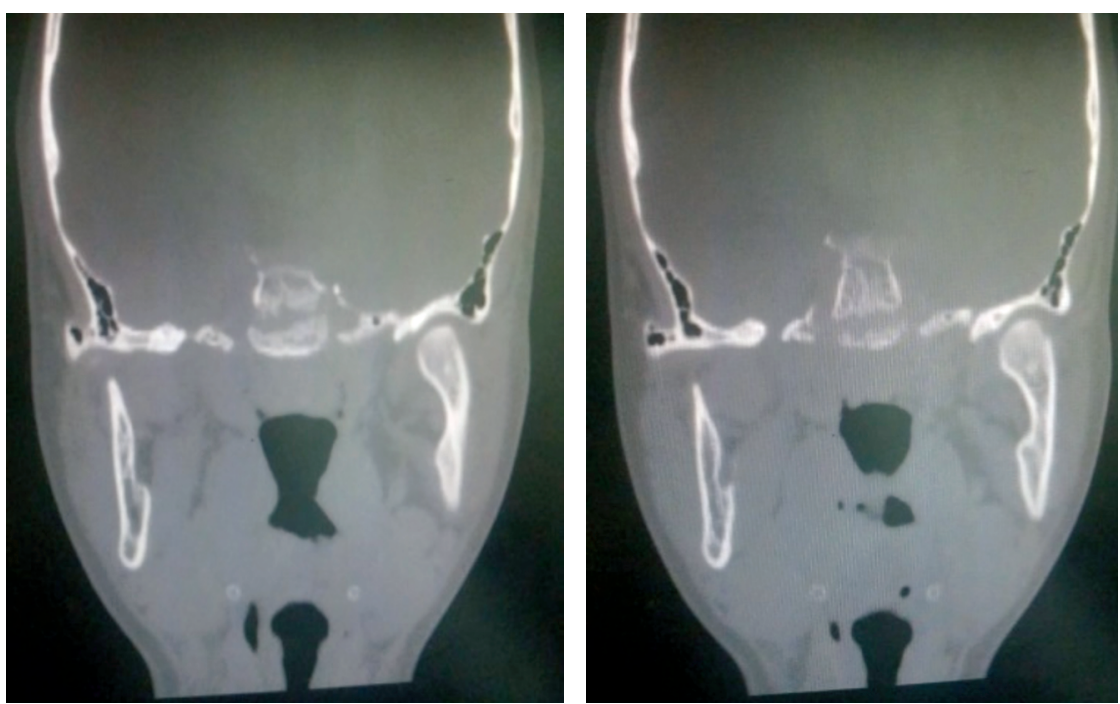

Fig. (3): A- CT facial (coronal, $3 \mathrm{D}$ view) of a 7 year old male patient with Lt subcondylar fracture and limited mouth opening, B- Upper and lower arch bar application with circum-mandibular circum-zygomatic wiring with intermaxillary elastic guiding, C- 1 year post-operative with good occlusion and full range of mouth opening that shows minor degree of deviation D- CT facial (coronal view) showing good bone alignment.

(D)

\section{DISCUSSION}

Direct trauma to the mandible can result in proximal transmission of force, leading to injury of the condylar process. This is in contrast to injuries in other areas of the mandible, which are often the result of direct trauma. Injuries involving the temporomandibular joint can result in growth disturbance in young, facial asymmetry, malocclusion, limited mouth opening, and bony ankylosis [3]. These potential complications make identifica- tion, appropriate treatment, and long-term followup of condylar fractures essential [19].

Botulinum neurotoxin (BTX) is produced by spore-forming bacterium Clostridium botulinum [20]. It exerts dose-dependent weakness in muscles by blocking the release of acetylcholine from presynaptic motor nerve endings [21]. The usual maximum total recommended therapeutic dose at an injection session is about $80-100 \mathrm{U}$. The human lethal dose is estimated to be approximately $3000 \mathrm{U}$. 
Thus, clinical dose can be given safely without a fatal overdose complication [22]. The clinical effects appear between one and three days after administration of BTX, and the maximum effects occur after one-two weeks, which then stabilize to a moderate level until complete recovery of the nerve in approximately 3 months [23]. In this study, 1020 units of (BTX) were used whereas in other studies [8], a suggested dose of 20-40 units per time. The dose of (BTX) is individualized to the patient and many factors should be considered such as muscle size and activity.

The Lateral Pterygoid (LPM), is a muscle involved in mastication and associated with mouth opening movements. It consists of two heads, where the lower head inserts into the neck of the mandibular condyle [24]. Accordingly the LPM was injected with BTX in this study to minimize its activity and keep the TMJ disc in a normal position at the early stage of treatment [25]. The paralysis of LPM will not disturb the passive mouth opening and mastication due to the assistance by the mylohyoid, geniohyoid and digastric muscles. The effect of (BTX) continues about 4 months which is parallel to the length of the time for a bone fracture to heal $[\mathbf{2 6 , 2 7 ]}$. Taking into consideration that the maxillary artery passes medial to the muscle or lateral to the lower head of LPM [28], the injection of BTX was ultrasound guided to avoid injury of maxillary artery and hence avoiding the risk of possible complications such as bleeding and hematoma.

Many studies showed that a condylar fracture leads to a complex sequence of alterations that try to restore an articulation to enable mastication. These adaptations, namely, neuromuscular, skeletal and dental adaptations begin immediately after injury but vary somewhat in their timing and importance. Neuromuscular adaptations are early, and short-term, that help in positioning the mandible until a new skeletal articulation has been obtained [29]. Based on these studies, our usage of (BTX) augments the early neuromuscular adaptations while decreasing, the pull off of the LPM on fracture site thus promoting quicker adaptation and better occlusion.

There are two major forces effective in the displacement of the fractured condylar segment: Traumatic force leading to fracture of the bone and contraction of masticatory muscles inserting to this segment. In other words, even if the fractured segment was not displaced by the effect of the traumatic force, it may still be displaced by the contraction of masticatory muscles. Therefore one must overcome the contractile forces of these muscles both for proper reduction of displaced segments and keeping them in reduced position [17]. Using (BTX) in this study supports very much this concept by antagonizing the force of the masticatory muscle, which, ends with keeping reduced segments in place and faster healing with decreased or no pain.

In this study, we adopted early mobilization of the jaw by intermaxillary guiding elastics and longterm functional therapy, it is in our believe that the less the MMF, the better TMJ function postoperative and the less invasive technique is chosen, the less the chance of running into complications. Our results are comparable with other reports [30,31]. Further studies with larger samples are necessary to determine the optimal treatment dosage.

\section{Conclusion:}

Based on the result of this study, the use of botulinium toxin in condylar fractures proves to be a safe and an adjuvant modality in managing these patients. However, more randomized, controlled, blinded clinical researches are needed to understand the properties, clinical efficacy, and any associated long-term adverse effects.

\section{REFERENCES}

1- Ellis E. and Throckmorton G.S.: Treatment of mandibular condylar process fractures: Biological considerations. J. Oral Maxillofac. Surg., 63: 115-34, 2005.

2- Tasanen A. and Lamberg M.A.: Trans-osseous wiring in the treatment of condylar fractures of the mandible. J. Maxillofac. Surg., 4: 200-6, 1976.

3- Ellis E. $3^{\text {rd }}$, Moos K.F. and El-Attar A.: Ten years of mandibular fractures: An analysis of 2,137 cases. Oral Surg. Oral Med. Oral Pathol., 59: 120-9, 1985.

4- Vural E.: Treatment of adult subcondylar mandibular fractures: Closed vs open vs endoscopic approach. Arch. Otolaryngol. Head Neck Surg., 130: 228-30, 2004.

5- Brandt M.T. and Haug R.H.: Open versus closed reduction of adult mandibular condyle fractures: A review of the literature regarding the evolution of current thoughts on management. J. Oral Maxillofac. Surg., 61: 1324-32, 2003.

6- Merlet F.L., Grimaud F., Pace R., Mercier J.M., Poisson M., Pare A. and Corre P.: Outcomes of functional treatment versus open reduction and internal fixation of condylar mandibular fracture with articular impact: A retrospective study of 83 adults, Journal of Stomatology, Oral and Maxillofacial Surgery, 119 (1): 8-15, 2018.

7- Rozeboom A.V.J., Dubois L., Bos R.R.M., Spijker R. and Lange J.D.: Closed treatment of unilateral mandibular condyle fractures in adults: A systematic review, International Journal of Oral and Maxillofacial Surgery, 46 (4): 456-64, 2017. 
8- Clark G.T.: The management of oromandibular motor disorders and facial spasms with injections of botulinum toxin. Phys. Med. Rehabil. Clin. N. Am., 14: 727-48, 2003.

9- Lang A.M.: Botulinum toxin therapy for myofascial pain disorders. Curr. Pain Headache Rep., 6: 355-60, 2002.

10- Freund B., Schwartz M. and Symington J.M.: Botulinum toxin: New treatment for temporomandibular disorders. Br. J. Oral Maxillofac. Surg., 38: 466-71, 2000.

11- Lovell B.V. and Marmura M.J.: New therapeutic developments in chronic migraine. Curr. Opin. Neurol., 23: 2548, 2010.

12- Bhogal P.S., Hutton A. and Monaghan A.: A review of the current uses of Botox for dentally-related procedures. Dent. Update, 33: 165-8, 2006.

13- Daelen B., Thorwirth V. and Koch A.: Treatment of recurrent dislocation of the temporomandibular joint with type A botulinum toxin. Int. J. Oral Maxillofac. Surg., 26: 458-60, 1997.

14- Moore A.P. and Wood G.D.: Medical treatment of recurrent temporomandibular joint dislocation using botulinum toxin A. Br. Dent. J., 183: 415-7, 1997.

15- Ziegler C.M., Haag C. and Mühling J.: Treatment of recurrent temporomandibular joint dislocation with intramuscular botulinum toxin injection. Clin. Oral Investig., 7: 52-5, 2003.

16- Fu K.Y., Chen H.M., Sun Z.P., Zhang Z.K. and Ma X.C.: Long-term efficacy of botulinum toxin type A for the treatment of habitual dislocation of the temporomandibular joint. Br. J. Oral Maxillofac. Surg., 48: 281-4, 2010.

17- Halil I.C., Aycan K., Muge A. and Mehmet E.M.: Botulinum Toxin in Closed Treatment of Mandibular Condylar Fracture, Annals of Plastic Surgery, 58 (5): 474-8, 2007.

18- Bbagol A., Singb V., Kumar I. and Verma A.: Prospective evaluation of a new classification system for the management of mandibular subcondylar fractures J. Oral MaxilLofac. Surg., 69: 1159-65, 2011.

19- Ghasemzadeh A., Mundinger G.S., Swanson E.W., Utria A.F. and Dorafshar A.H.: Treatment of Pediatric Condylar Fractures: A 20-Year Experience, Plast. and Reconstr. Surg., 136 (6): 1278-88, 2015.
20- Welch M.J., Purkiss J.R. and Foster K.A.: Sensitivity of embryonic rat dorsal root ganglia neurons to Clostridium botulinum neurotoxins. Toxicon, 38 (8): 245-58, 2000.

21- Lu D.W. and Lippitz J.: Complications of botulinum neurotoxin. Dis. Mon., 55 (4): 198-211, 2009.

22- Schames J., Dov Prero Y., Schames D., Schames M. Gabriel W. and Reed R.: Uncontrollable distant effects of botulinum neurotoxin injections. J. Calif. Dent. Assoc., 37: 44-5, 2009.

23- Blitzer A. and Sulica L.: Botulinum toxin: Basic science and clinical uses in otolaryngology. Laryngoscope, 111 (2): 218-26, 2001.

24- Murray G.M., Orfanos T., Chan J.Y., Wanigaratne K. and Klineberg I.J.: Electromyographic activity of the human lateral pterygoid muscle during contralateral and protrusive jaw movements. Arch. Oral Biol., 44: 269-85, 1999.

25- Murray G.M., Bhutada M., Peck C.C., Phanachet I., SaeLee D. and Whittle T.: The human lateral pterygoid muscle. Arch. Oral Boil., 52: 377-80, 2007.

26- Aoki K.R. and Guyer B.: Botulinum toxin type A and other botulinum toxin serotypes: A comparative review of biochemical and pharmacological actions. Eur. J. Neurol., 8: 21-9, 2001.

27- Teixeira A.C., Luz J.G., Araujo V.C. and Araujo N.S.: Healing of the displaced condylar process fracture: An experimental study. J. Craniomaxillofac. Surg., 26: 32630, 1998.

28- Takarada T.: Anatomical studies on the maxillary artery. J. Tokyo Dent. Coll. Soc., 53: 1-20, 1958.

29- Talwar R.M., Ellis E. $3^{\text {rd }}$ and Throckmorton G.S.: Adaptations of the masticatory system after bilateral fractures of the mandibular condylar process. J. Oral Maxillofac. Surg., 56: 430-9, 1998.

30- Villarreal P.M., Monje F., Junquera L.M., et al.: Mandibular condyle fractures: Determinants of treatment and outcome. J. Oral Maxillofac. Surg., 62: 155-63, 2004.

31- Lindahl L.: Condylar fractures of the mandible, I: Classification and relation to age, occlusion, and concomitant injuries of teeth and teeth supporting structures, and fractures of the mandibular body. Int. J. Oral Surg., 6: 12-21, 1977. 